NASA/CR-2004-213200

\title{
Computational Study of Primary Electrons in the Cusp Region of an Ion Engine's Discharge Chamber
}

Shirin S. Deshpande, Sudhakar Mahalingam, and James A. Menart Wright State University, Dayton, Ohio 
Since its founding, NASA has been dedicated to the advancement of aeronautics and space science. The NASA Scientific and Technical Information (STI) Program Office plays a key part in helping NASA maintain this important role.

The NASA STI Program Office is operated by Langley Research Center, the Lead Center for NASA's scientific and technical information. The NASA STI Program Office provides access to the NASA STI Database, the largest collection of aeronautical and space science STI in the world. The Program Office is also NASA's institutional mechanism for disseminating the results of its research and development activities. These results are published by NASA in the NASA STI Report Series, which includes the following report types:

- $\quad$ TECHNICAL PUBLICATION. Reports of completed research or a major significant phase of research that present the results of NASA programs and include extensive data or theoretical analysis. Includes compilations of significant scientific and technical data and information deemed to be of continuing reference value. NASA's counterpart of peerreviewed formal professional papers but has less stringent limitations on manuscript length and extent of graphic presentations.

- TECHNICAL MEMORANDUM. Scientific and technical findings that are preliminary or of specialized interest, e.g., quick release reports, working papers, and bibliographies that contain minimal annotation. Does not contain extensive analysis.

- CONTRACTOR REPORT. Scientific and technical findings by NASA-sponsored contractors and grantees.
- CONFERENCE PUBLICATION. Collected papers from scientific and technical conferences, symposia, seminars, or other meetings sponsored or cosponsored by NASA.

- SPECIAL PUBLICATION. Scientific, technical, or historical information from NASA programs, projects, and missions, often concerned with subjects having substantial public interest.

- TECHNICAL TRANSLATION. Englishlanguage translations of foreign scientific and technical material pertinent to NASA's mission.

Specialized services that complement the STI Program Office's diverse offerings include creating custom thesauri, building customized databases, organizing and publishing research results ... even providing videos.

For more information about the NASA STI Program Office, see the following:

- Access the NASA STI Program Home Page at http://www.sti.nasa.gov

- E-mail your question via the Internet to help@sti.nasa.gov

- Fax your question to the NASA Access Help Desk at 301-621-0134

- Telephone the NASA Access Help Desk at 301-621-0390

- Write to:

NASA Access Help Desk

NASA Center for AeroSpace Information 7121 Standard Drive

Hanover, MD 21076 
NASA/CR-2004-213200

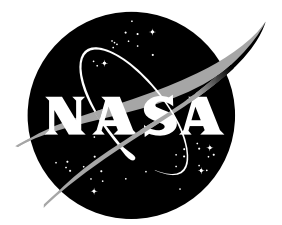

\section{Computational Study of Primary Electrons in the Cusp Region of an Ion Engine's Discharge Chamber}

Shirin S. Deshpande, Sudhakar Mahalingam, and James A. Menart Wright State University, Dayton, Ohio

Prepared for the 40th Joint Propulsion Conference and Exhibit cosponsored by the AIAA, ASME, SAE, and ASEE Fort Lauderdale, Florida, July 11-14, 2004

Prepared under Grant NAG3-2907

National Aeronautics and Space Administration

Glenn Research Center 


\section{Acknowledgments}

The authors would like to thank the NASA Glenn Research Center and grant monitors Michael Patterson and Thomas Steuber for financially supporting this work.

Available from

NASA Center for Aerospace Information 7121 Standard Drive

Hanover, MD 21076
National Technical Information Service 5285 Port Royal Road Springfield, VA 22100

Available electronically at http:/ /gltrs.grc.nasa.gov 


\title{
Computational Study of Primary Electrons in the Cusp Region of an Ion Engine's Discharge Chamber
}

\author{
Shirin S. Deshpande, Sudhakar Mahalingan, and James A. Menart \\ Wright State University \\ Dayton, Ohio 45435-0002
}

\begin{abstract}
In this work a computer code called PRIMA is used to study the motion of primary electrons in the magnetic cusp region of the discharge chamber of an ion engine. Even though the amount of wall area covered by the cusps is very small, the cusp regions are important because prior computational analyses have indicated that most primary electrons leave the discharge chamber through the cusps. The analysis presented here focuses on the cusp region only. The affects of the shape and size of the cusp region on primary electron travel are studied as well as the angle and location at which the electron enters the cusp region. These affects are quantified using the confinement length and the number density distributions of the primary electrons. In addition to these results comparisons of the results from PRIMA are made to experimental results for a cylindrical discharge chamber with two magnetic rings. These comparisons indicate the validity of the computer code called PRIMA.
\end{abstract}

\section{Nomenclature}

$\begin{array}{ll}A_{\theta} & =\text { Magnetic vector potential in circumferential direction } \\ \mu & =\text { Permeability in respective direction } \\ V & =\text { Velocity component in respective direction } \\ M_{\theta} & =\text { Angular momentum } \\ D & =\text { Diameter of discharge chamber } \\ \dot{m} & =\text { Propellant mass flow rate } \\ \eta & =\text { Propellant utilization efficiency } \\ n_{n} & =\text { Number density of the neutral particle } \\ \phi & =\text { Grid transparency } \\ A_{g} & =\text { Area of grid } \\ \ell_{a v e}^{*} & =\text { Normalized average confinement length } \\ n_{p e}^{*} & =\text { Normalized primary electron number density } \\ \sigma_{o} & =\text { Total inelastic collision cross section }\end{array}$

\section{Introduction}

In order to effectively understand the workings of the discharge chamber of the ion engine a greater knowledge of how primary electrons behave in the cusp region must be obtained. Computational work done by Arakawa and Yamada, Arakawa and Ishihara, and Mahalingam and Menart show primary electron trajectory plots that indicate that most primary electrons leave the discharge chamber through the cusps. ${ }^{1,2,3}$ Taken from the work of Mahalingam and Menart with permission is a trajectory plot of a primary electron in a conical-cylindrical discharge chamber with two permanent magnetic rings (Fig. 1). ${ }^{3}$ This trajectory plot shows one primary electron getting close to the anodebiased wall only in the cusp region. For this reason it is important to understand how the shape and strength of these cusps affect the primary electrons. This computational study is an effort to add to this knowledge. 
The only work known to the authors that has specifically investigated the cusp region of an ion thruster is that of Hirakawa and Arakawa. ${ }^{4}$ Hirakawa and Arakawa's model includes magnetic as well as electric field effects in a two dimensional, rectangular region around a line-cusp. One of the major goals of Hirakawa and Arakawa was to study the ion loss mechanism. ${ }^{4}$ In the work presented here the focus will be solely on the primary electrons in a cylindrical, axisymmetric, ring-cusp configuration and electric fields will not be included. However, this work will include the effects of particle collisions; whereas Hirakawa and Arakawa ignored this phenomenon. ${ }^{4}$ They may have ignored this because their computational domain was extremely small. In this work a much larger portion of

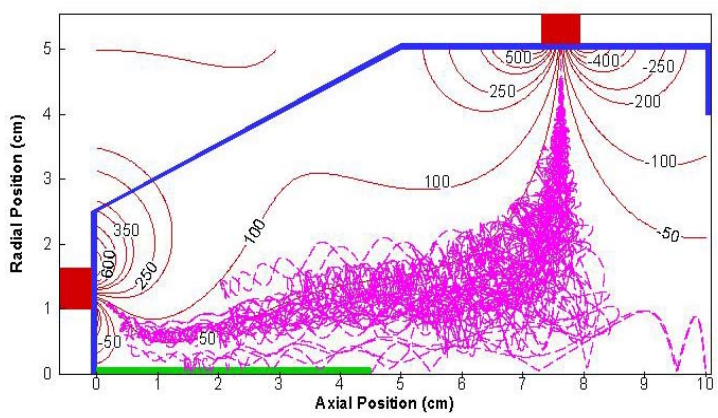

Figure 1. Path of a primary electron in a conical cylindrical ring - cusp discharge chamber the cusp will be studied. Particle collisions are deemed to be important because they can force charged particles to cross strong magnetic field lines. ${ }^{3}$

In addition to the above stated differences to Hirakawa and Arakawa's work another is the magnetic field used. In this work magnetic fields produced by permanent magnets will be determined numerically. Hirakawa and Arakawa $^{4}$ used an analytical expression taken from the work of Samec. ${ }^{5}$ This work will calculate the fields produced by permanent magnets by solving Maxwell's equations. ${ }^{6}$

Other efforts to study the cusp region in regards to magnetic field confinement can be found in other plasma disciplines besides ion engines. Three of these works are Marcus et al. ${ }^{7}$, Leung et al. ${ }^{8}$, and Bosch and Berlino. ${ }^{9}$

The computer codes utilized to perform this study are MAXWELL 2D and PRIMA. MAXWELL 2D is a commercial code for solving magnetic fields in two-dimensional Cartesian or cylindrical coordinates. ${ }^{10}$ PRIMA is essentially a particle-in-cell (PIC) code that tracks primary electrons around the computational domain. This code was originally developed by Arakawa and Yamada. ${ }^{2}$ Recently Mahalingam and Menart have upgraded this particular code. ${ }^{3}$ This upgraded version of PRIMA is used in this work.

\section{Mathematical Model}

There are two major parts of the mathematical model used in this work: one is the modeling of the magnetic field and the other is the modeling of the primary electron trajectories. First, a brief discussion of the magnetic field modeling will be given and then a brief discussion of the primary electron modeling will be given. More details on the magnetic field code MAXWELL 2D can be found at the Ansoft Corporation web site ${ }^{10}$ and details for the computer code PRIMA can be found in Mahalingam and Menart. ${ }^{3}$

\section{A. Magnetic Field Modeling}

The steady state magnetic field produced by permanent magnet rings in a two-dimensional, axisymmetric situation with no free currents can be determined by solving a subset of Maxwell's equations. The governing equation to determine the magnetic field is

$$
\frac{\partial}{\partial r}\left(\frac{1}{r \mu_{z}} \frac{\partial\left(r A_{\theta}\right)}{\partial r}\right)+\frac{\partial}{\partial z}\left(\frac{1}{\mu_{r}} \frac{\partial A_{\theta}}{\partial z}\right)=\frac{\partial H_{c r}}{\partial z}-\frac{\partial H_{c z}}{\partial r}
$$

where $\mathrm{r}$ is the radial coordinate and $\mathrm{z}$ is the axial coordinate. The quantity $A_{\theta}$ is the magnetic vector potential in the circumferential direction. In axisymmetric situations the other components of the magnetic vector potential are not required. This is the beauty of the magnetic vector potential. The circumferential component of the magnetic vector potential is related to the radial, $B_{r}$, and axial, $B_{z}$, components of the magnetic field by 


$$
\begin{gathered}
B_{r}=\frac{1}{r} \frac{\partial\left(r A_{\theta}\right)}{\partial r} \\
B_{z}=\frac{\partial A_{\theta}}{\partial z}
\end{gathered}
$$

The quantities $H_{c r}$ and $H_{c z}$ are the coercive force of the permanent magnets in the radial and axial directions respectively. Equation (1) is solved over a fairly large computational domain. Essentially the computational domain is made big enough such that the following Dirichlet boundary conditions can be used:

$$
\begin{aligned}
& A_{\theta} \rightarrow 0 \text { as } \quad r \rightarrow \infty \\
& A_{\theta} \rightarrow 0 \text { as } \quad z \rightarrow-\infty \\
& A_{\theta} \rightarrow 0 \text { as } \quad z \rightarrow \infty
\end{aligned}
$$

Since the computation is being done for ring cusp magnets, the kind used on NASA's $30 \mathrm{~cm}$ ion engine ${ }^{11}$ the fourth boundary condition required is for an axis of symmetry. This boundary condition is a Neumann boundary condition

$$
\frac{\partial A_{\theta}}{\partial r}=0 \quad \text { at } \quad r=0
$$

In this work the magnetic field is calculated for the magnet configuration shown in Fig. 2. This configuration is three magnetic rings located on an aluminum wall that is $1 \mathrm{~mm}$ thick. The aluminum wall does not interact with the magnetic field but it does move the location where the primary electrons are absorbed out from the magnet surface by $1 \mathrm{~mm}$. The inside radius of the discharge chamber wall is taken to be $5 \mathrm{~cm}$. The cusp used for the detailed primary electron calculation is the center cusp. Three magnet rings are used to produce the cusp region so that the cusp is symmetric. The strength of the cusp is altered by changing the strength of the permanent magnets used. The shape of the cusp region is changed by altering the spacing, $\mathrm{z}_{\mathrm{m}}$, between the magnets (Fig. 2). When the spacing is changed, the strength of the cusp region changes some also.

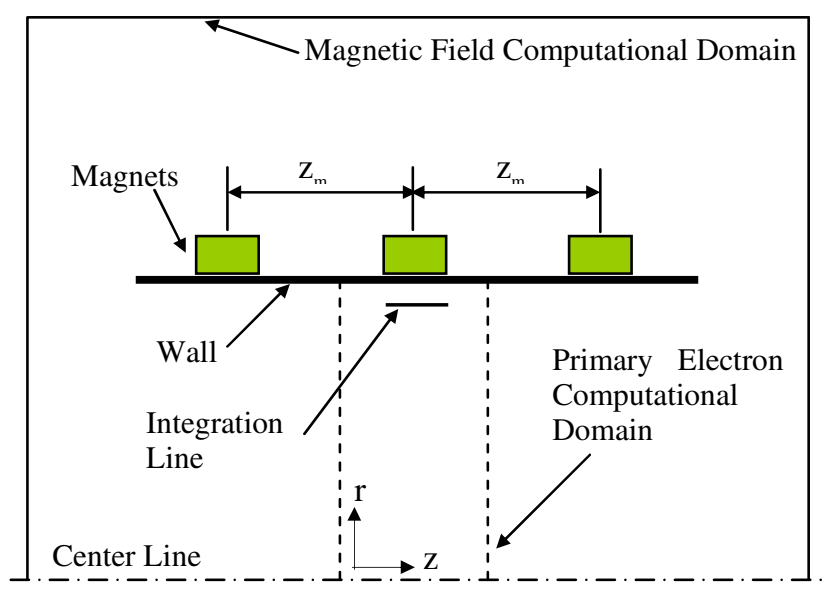

Figure 2. Computational domains used for magnetic field calculation and the primary electron calculation.

\section{B. Primary Electron Model}

The primary electron computational domain is much smaller than that for the magnetic field in order to focus on the phenomenon happening in the cusp region (Fig. 2). The primary electron computational domain is $4 \mathrm{~cm}$ long in the axial direction and $5 \mathrm{~cm}$ high in the radial direction. The computational domain is centered on the middle magnet. For most of the results presented in this paper the primary electrons are emitted from the lower left corner of the primary electron computational domain, and are emitted in all directions into the computational domain. To study the effect of the location where the primary electrons are emitted, results will be presented for the electrons being emitted from two different locations along the center line. 
The equations of motion for a primary electron for an axisymmetric geometry were developed from the EulerLagrange equations utilizing a Lagrangian written for a charged particle traveling in a magnetic field. ${ }^{12,13}$ In nondimensional form these equations are

$$
\begin{gathered}
\frac{d V_{r}^{*}}{d t^{*}}=\frac{V_{\theta}^{* 2}}{r^{*}}-\frac{V_{\theta}^{*}}{r^{*}} \frac{\partial\left(r^{*} A_{\theta}^{*}\right)}{\partial r^{*}} \\
\frac{d V_{z}^{*}}{d t^{*}}=-V_{\theta}^{*} \frac{\partial A_{\theta}^{*}}{\partial z^{*}} \\
M_{\theta}^{*}=r^{*} V_{\theta}^{*}-r^{*} A_{\theta}^{*}
\end{gathered}
$$

where the superscript $*$ represents a nondimensional quantity. In these expressions $t$ is time and $V$ is velocity in the radial, $r$, axial, $z$, and circumferential, $\theta$, directions. The quantity $M_{\theta}^{*}$ is a conserved quantity in the circumferential direction until the electron undergoes a collision. The reference quantities used to nondimensionalize the above quantities are:

$$
\begin{gathered}
r_{r e f}=D \text { And } z_{r e f}=D \\
V_{r e f}=\sqrt{\frac{2|e| V_{d}}{m}} \\
V_{r, r e f}, V_{\theta, r e f}, \text { and } V_{z, r e f}=V_{r e f} \\
t_{r e f}=\frac{D}{V_{r e f}} \\
A_{\theta, r e f}=\frac{m V_{r e f}}{e} \\
B_{r e f}=\frac{m V_{r e f}}{D|e|} \\
M_{\theta, r e f}=m D V_{r e f}
\end{gathered}
$$

In these equations $D$ is twice the radius of the wall (Fig. 2), $V_{d}$ is the discharge voltage, $m$ is the mass of an electron, and $|e|$ is the magnitude of the electric charge on an electron.

The wall collisions considered in this model are of the elastic type except for collisions with the anode-biased wall. When the primary electron collides with the cusp wall it is absorbed and the calculation for another primary electron begins. This means that the total energy of the primary electron remains the same until it is removed from the computational domain by a collision with the cusp wall. The cusp wall absorbs primary electrons along its entire length. At the non-cusp boundaries of the primary electron computational domain the primary electrons are reflected back into the computational domain.

The elastic particle collisions considered in this analysis are: 1) primary electrons with neutral atoms, 2) primary electrons with singly charged positive ions, and 3) primary electrons with other electrons. While the computer code used to perform these calculations can handle ionizing collisions with neutral particles these are not included in the 
analysis. Ionizing collisions have the effect of changing a primary electron into a secondary electron. Since ionizing collisions are the purpose for which primary electrons were produced they are desirable. Therefore it is not desired that ionizing collisions be detrimental to the value calculated for the electron confinement length. Including ionizing collisions shortens the electron confinement length indicating a poor cusp design, even though something desirable is happening. Brophy and Wilbur state that inelastic collisions should not be included in the determination of the confinement length. ${ }^{14}$ In order to determine the number of elastic collisions in the cusp region the neutral atom density, $n_{n}$, is calculated from the propellant flow rate, $\dot{m}$, a propellant utilization efficiency, $\eta_{\text {, the }}$ area of the grids, $A_{g}$, the velocity of neutral particles, $V_{n}$, and the transparency of the grids to neutral particles, $\phi_{n}$,

$$
n_{n}=\frac{4 \dot{m}(1-\eta)}{|e| V_{n} \phi_{n} A_{g}}
$$

and that is input into the program. Ion number densities, $n_{i}$, are calculated from

$$
n_{i}=\frac{\dot{m} \eta}{|e| V_{i} \phi_{i} A_{g}}
$$

where $\phi_{i}$ is the transparency of the grids to the ions, $A_{g}$ is the area of the grids, and $V_{i}$ is the velocity of ions determined from the Bohm criteria. The overall electron number densities, $n_{e}$, are set equal to the ion number density, $n_{i}$ Xenon is the gas used in all results shown except for the experimental comparisons where argon is used.

The confinement length for a single primary electron is nothing more than the distance traveled by that electron during its lifetime. The confinement length for a cusp region is defined here as the average of a very large number of primary electrons that enter the cusp region. ${ }^{3}$ In theory the number of primary electrons should be very large; however, in practice only a finite number of primary electrons have to be tracked to determine this value. Because of the nondimensionalization chosen for the velocities, the magnitude of the primary electron's velocity is 1.0 during its entire lifetime. This means the nondimensional confinement length for an individual particle is exactly equal to the nondimensional time it spends in the discharge chamber. Thus the nondimensional cusp confinement length, $\ell_{\text {ave }}^{*}$, can be determined from

$$
\ell_{\text {ave }}^{*}=\frac{1}{N} \sum_{j=1}^{N} t_{\text {conf }, j}^{*}
$$

where $N$ is the total number of primary electrons tracked throughout the discharge chamber and $t_{\text {conf }, j}^{*}$ is the nondimensional confinement time for each individual primary electron. The dimensional confinement length can be determined by multiplying the nondimensional confinement length by the discharge chamber diameter.

The other output quantity from this analysis is the relative primary electron number density, $n_{p e}^{*}$

$$
n_{p e}^{*}=\frac{n_{p e}}{n_{p e, \max }}
$$

where $n_{p e}$ is the primary electron number density at some location and $n_{p e, \max }$ is the maximum primary electron number density found in the cusp region. The relative primary electron number density is determined from

$$
n_{p e}^{*}=\frac{\sum_{j=1}^{N} \Delta t_{\text {element }, j}^{*}}{\left[\sum_{j=1}^{N} \Delta t_{\text {element }, j}^{*}\right]_{\max }}
$$


where $\Delta t_{\text {element }, j}^{*}$ is the total time spent by an individual primary electron in one of the finite elements at a given location. The denominator represents the finite element where primary electrons reside the most. This is generally close to the exit of the cathode.

\section{Experimental Comparison}

In order to gain some confidence in the numerical results produced by PRIMA a comparison to the experimental results of Hiatt and Wilbur ${ }^{15}$ are made. The case compared to is a two ring, straight cylindrical discharge chamber that has a chamber diameter of $9.2 \mathrm{~cm}$ and a beam diameter of $8.0 \mathrm{~cm}$. The discharge chamber is similar to the one shown in Fig. 1 except there is no slanted wall, the magnets are placed inside the steel walls, and the magnet on the back wall is placed right on the centerline. The only place in the discharge chamber at which electrons can be collected is at the cusp of the magnet located on the side wall of the discharge chamber. All of the walls of the discharge chamber are biased at the cathode potential. The second magnetic ring was located on the upstream wall of the discharge chamber. The cathode used was a hot filament made of small diameter tungsten wire and formed into the shape of a 1 to $6 \mathrm{~cm}$ diameter loop. The cathode filament is located downstream of the cusp of the side-wall magnet close to the grids

The main parameter that was compared is the primary electron utilization factor. This quantity is determined with the equation

$$
C_{o}=\frac{4 \sigma_{o} \ell_{a v e}^{*} D}{|e| V_{n} \phi_{o} A_{g}}
$$

where $\sigma_{o}$ is the total inelastic collision cross-section, $\ell_{\text {ave }}^{*}$ is the average confinement length, $D$ is the diameter of the discharge chamber, $V_{n}$ is the average velocity of the neutral particles, $\phi_{o}$ is the grid transparency to neutral atoms, and $A_{g}$ is the area of the grids. The quantity in this equation that is determined by the computer code PRIMA is $\ell_{\text {ave }}^{*}$. Thus good comparisons of $C_{o}$ indicate that PRIMA is producing reasonable results for $\ell_{\text {ave }}^{*}$.

The comparisons between the computation results and the experimental results of Hiatt and Wilbur ${ }^{15}$ are shown in Table 1. Hiatt and Wilbur obtained a value of $4.5(\mathrm{amp} \mathrm{eq})^{-1}$ for their anode on the cusp discharge chamber configuration. This discharge configuration was used for many different propellant mass flow rates and propellant utilization parameters. From this set of data they were able to plot a plasma ion energy cost versus the neutral number density parameter, $m(1-\eta)$. Ideally, and for the most part practically, $C_{o}$ does not depend on the neutral number density parameter. This is the reason there is only one $C_{o}$ value listed for Hiatt and Wilbur's experimental results. If the individual measured results are viewed in Hiatt and Wilbur's paper some fluctuation with $m(1-\eta)$ will be seen, but it is small except at values of the neutral number

Table 1. Comparisons of $C_{o}$ values from PRIMA to an experimentally determined $C_{o}$ value.

\begin{tabular}{|c|c|c|c|}
\hline $\begin{array}{c}m(1-\eta) \\
(\text { amp eq. })^{-1}\end{array}$ & $\begin{array}{l}\text { This work } \\
C_{o} \\
\text { (amp eq.) }\end{array}$ & $\begin{array}{l}\text { Exp. } C_{o} \\
(\text { amp eq. })^{-1}\end{array}$ & $\begin{array}{c}\% \\
\text { Diff. }\end{array}$ \\
\hline 0.040 & 6.35 & \multirow[t]{4}{*}{4.5} & 41 \\
\hline 0.263 & 4.62 & & 3 \\
\hline 0.476 & 4.12 & & 8 \\
\hline 0.581 & 3.91 & & 13 \\
\hline
\end{tabular}
density parameter below 0.120 (amp eq) ${ }^{-1}$.

PRIMA was run at four different neutral number density parameters for this discharge chamber configuration. From the results in Table 1 it can be seen that the calculated $C_{o}$ value decreases slightly with an increase in the neutral number density parameter. Since all the other parameters in the $C_{o}$ equation, Eq. (23), are constant, $C_{o}$ must go down as the confinement length goes down. In general the comparisons shown in Table 1 are very good. The one comparison that is poor is the first one listed in the table. This point is located on the steep portion of the plasma ion energy cost versus the neutral number density parameter curve. Even Wilbur's data deviates from the fitted curve using $C_{o}=4.5(\mathrm{amp} \mathrm{eq})^{-1}$. The data deviates substantial for a neutral number density parameter less than 0.120 (amp $\mathrm{eq})^{-1}$. For these values, utilizing the same baseline ion production cost, a $C_{o}$ value closer to $6.6(\mathrm{amp} \text { eq })^{-1}$ is 
probably more reasonable for Hiatt and Wilbur's experimental data. This is within $4 \%$ of the numerical value calculated in this work.

\section{Convergence Study}

Because the gradients of the magnetic fields are extremely large in the cusp region it is necessary to perform a convergence study. As part of this work a number of numerical quantities are checked for convergence. These quantities are the computational domain size and the element size used for the magnetic field calculation, and the element size, the upper, $\Delta t_{\text {upper }}^{*}$, and lower, $\Delta t_{\text {lower }}^{*}$, limit on the internally calculated time step, and the number of primary electrons used in the primary electron calculation, $N$.

All results in this paper, except those for which another quantity is specified, use permanent magnets that have a magnetic coercive force of -9300 Oersted and a magnetic residual of 10000 Gauss. The size of the magnets used to produce the magnet rings is $0.635 \mathrm{~cm}$ in the axial direction and $0.475 \mathrm{~cm}$ in the radial direction. The thickness of the aluminum wall is taken to be $0.1 \mathrm{~cm}$. The mass flow rate was taken to be 0.200 Amp eq., the propellant utilization was taken as 0.8 , and the grid transparency to ions was 0.4 . The grid transparency to neutrals was 0.1 , and the discharge chamber voltage was 30 volts. The standard magnetic spacing was $7 \mathrm{~cm}$.

The convergence study performed with MAXWELL 2D showed that the magnetic field computational domain should be $15 \mathrm{~cm}$ high in the radial direction and the two side boundaries should be $13 \mathrm{~cm}$ away from the outer magnets (Fig. 2). The primary concern for the magnetic field was that it was converged in the primary electron computational domain. The number of finite elements required for the magnetic field to be converged was determined to be 9000 triangular elements. The fields for all the results presented in this paper are produced using permanent magnets. For the strength study the fields produced with the full strength magnets are reduced or enlarged by some factor.

Convergence studies for the primary electrons are carried out on a $5 \mathrm{~cm}$ (radial direction) by $4 \mathrm{~cm}$ (axial direction) primary electron computational domain using the magnetic fields produced by 3 permanent magnets separated by $7 \mathrm{~cm}$. The output quantity used to determine whether a solution is converged in terms of the primary electron calculation is the nondimensional confinement length. Convergence of the nondimensional confinement length will not guarantee convergence at all locations for the relative number density. In regions where the relative number density is small convergence will not be obtained. To obtain a converged relative number density at all locations in the cusp region is not possible from a practical perspective due to limited computational

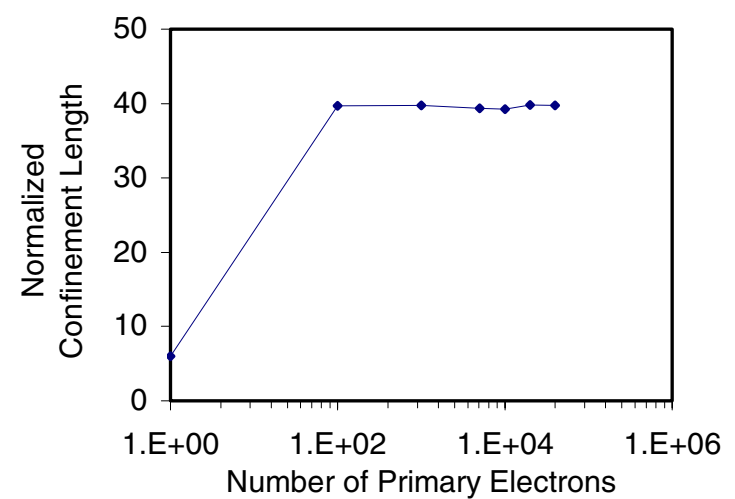

Figure 3. Convergence of number of primary electrons used in PRIMA computation.

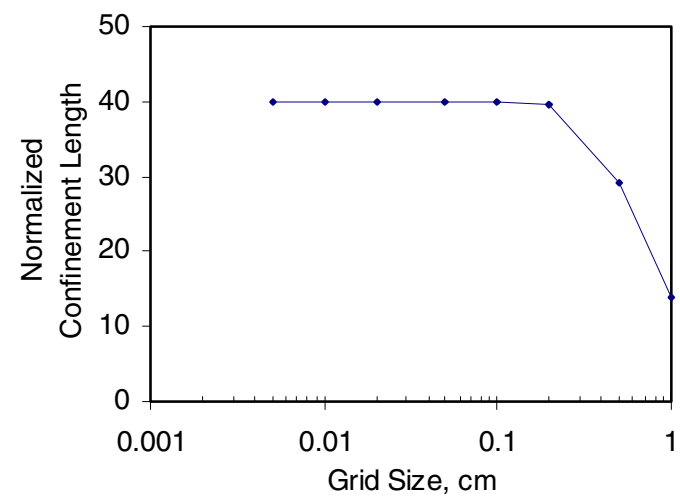

Figure 4. Convergence of grid size used in PRIMA computation.

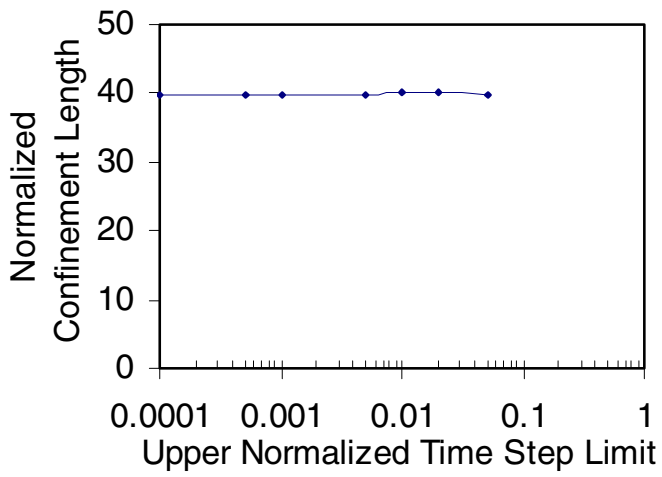

Figure 5. Convergence of upper limit of time step size used in PRIMA computation 
resources. Even with parallelization of PRIMA computational run times are long. Regions with small relative number densities will have to be viewed as being small but imprecise.

The first convergence study carried out for the operation of the computer program PRIMA is the number of primary electrons used in the computation. The results for this convergence study are shown in Fig. 3. As can be seen from this plot reasonable confinement lengths are obtained with as little as 100 particles. However, in order to converge a greater portion of the relative number density distributions 20,000 particles are used in the present computation. All cases do not show convergence of the normalized confinement length at such a low number of particles.

The results of the convergence study for the finite element size are shown in Fig. 4. The size of the rectangular elements is taken to be the same in the $r$ and $z$ directions. Convergence was taken to be with an element size of $0.02 \mathrm{~cm}$ by $0.02 \mathrm{~cm}$.

The results of the convergence study for the time step size are shown in Fig. 5,6. There are two different time steps used in PRIMA. PRIMA essentially determines its own time step size based on the strength of the magnetic field present at the particle location. However, the user of PRIMA puts a lower and upper limit on the size of the time step. The upper limit is chosen such that PRIMA does not pick too big of a time step and the lower limit is picked so that PRIMA does not pick too small of a time step. The reason for not picking too large of a time step is accuracy and the reason for not picking too small of a time step is computational time. As can be seen from Fig. 5 the upper limit on the time step is not important. This means PRIMA is utilizing very small time steps in the numerical calculation. The value used for the upper limit on the time step in this work is 0.001 . This is a normalized time step size. As Fig. 6 shows the lower limit on the time step is important. For this work a normalized value of $5 \times 10^{-5}$ is chosen. All the convergence parameters chosen in this study converge the normalized confinement to within $2 \%$ of the best value calculated.

\section{Survey Results}

\section{A. Magnetic Field Shape}

In order to change the shape of the cusp region the separation of the magnets, $z_{m}$, is increased. The magnet separation distance is varied from $3 \mathrm{~cm}$ to $15 \mathrm{~cm}$. The magnetic field lines for the $3 \mathrm{~cm}$ separation distance are shown in Fig. 7 and the magnetic field lines for the $15 \mathrm{~cm}$ case are shown in Fig. 8. As can be seen in these figures

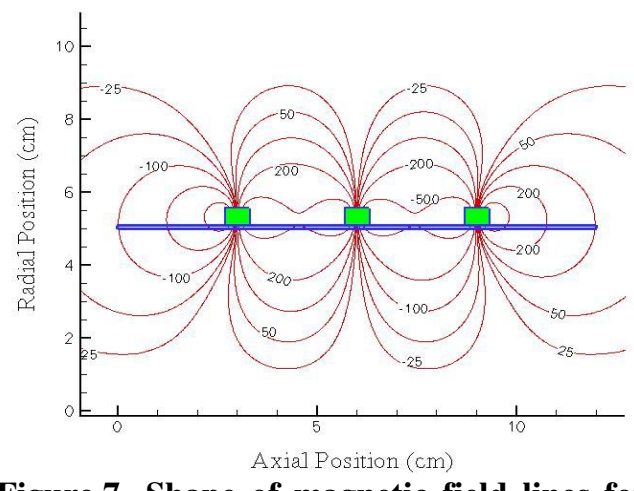

Figure 7. Shape of magnetic field lines for magnet spacing of $3 \mathrm{~cm}$. These are shown for magnetic field computational domain

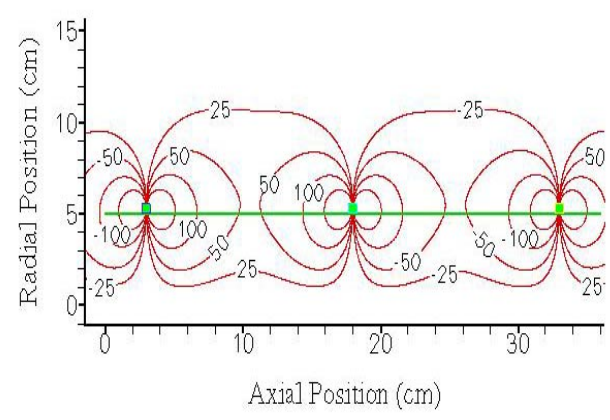

Figure 8. Shape of magnetic field lines for magnet spacing of $15 \mathrm{~cm}$. These are shown for magnetic field computational domain.

different types of cusp shapes are obtained. When the magnets are very close together, $z_{m}=3 \mathrm{~cm}$, most of the field lines originate on the center magnet and terminate on the adjacent magnets. When the magnets are far apart a large number of field lines originate on one side of the center magnet and terminate on the other side of the center magnet. 
The two side magnets are too far away and the field lines prefer to go around to the backside of the same magnet. Magnet spacing between 3 and $15 \mathrm{~cm}$ provide some intermediate type of field shape to that shown in Fig. 7,8. The magnetic flux density contours for just the primary electron computational domain are shown in Fig. 9,10 for magnet spacing of 3 and $15 \mathrm{~cm}$. These plots are also different, but care must be taken in comparing them.

The effect of the spacing between the magnets is shown in Fig. 11. This figure displays a somewhat surprising

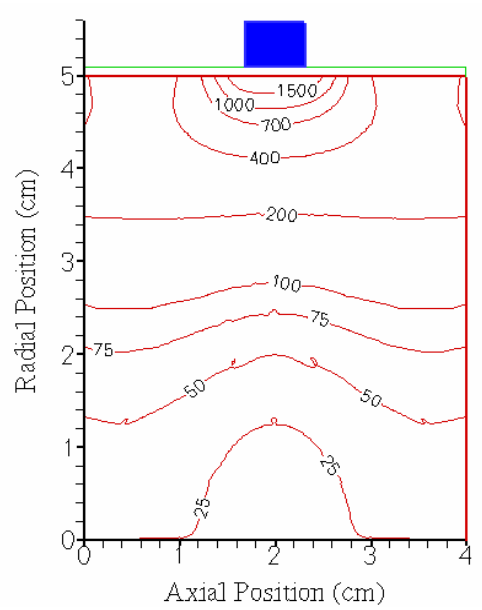

Figure 9. Magnetic flux density contours for a magnetic spacing of $3 \mathrm{~cm}$. These are shown for the primary electron computational domain only.

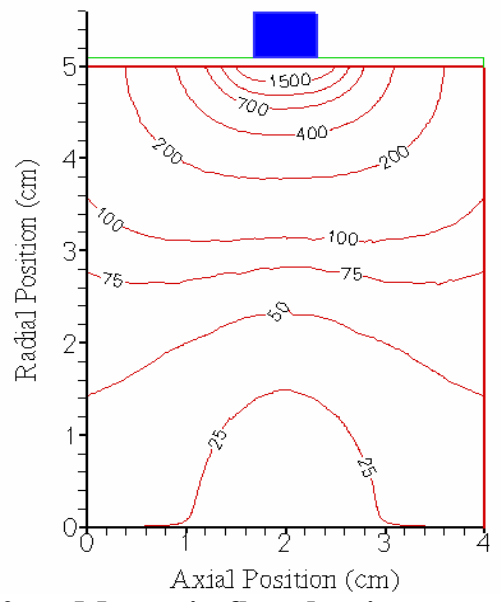

Figure 10. Magnetic flux density contours for a magnetic spacing of $15 \mathrm{~cm}$. These are shown for the primary electron computational domain only.

result; the confinement length is a minimum value for intermediate magnet spacing distances. In this plot the $3 \mathrm{~cm}$ magnet spacing produces a normalized confinement length of 87 , the $15 \mathrm{~cm}$ spacing a value of 63 , and the minimum value occurs at $5 \mathrm{~cm}$ spacing with a value of 39. This means the mirroring ability of the cusp; its ability to reflect particles back out, depends on its shape.

It must be realized that this study only considers a primary electron computational domain right around the cusp. This means that weak magnetic fields between magnets are not included. Even though a $15 \mathrm{~cm}$ magnetic spacing produces a longer confinement length than the $5 \mathrm{~cm}$ magnet spacing this does not mean that this will be a better magnet spacing for an actual discharge chamber. It is planned to study the overall effect of magnet spacing in future work.

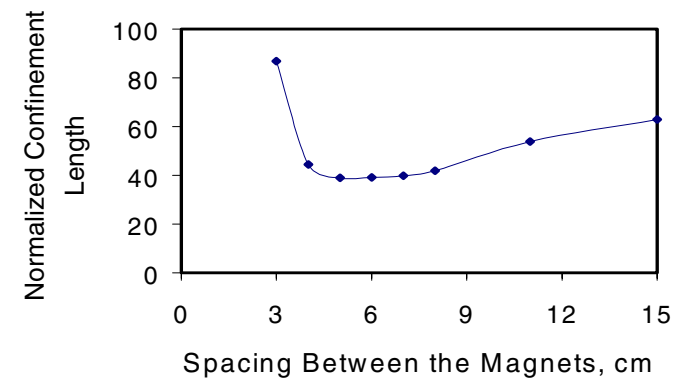

Figure 11. Effect of magnetic field shape displayed as function of the spacing between the magnets

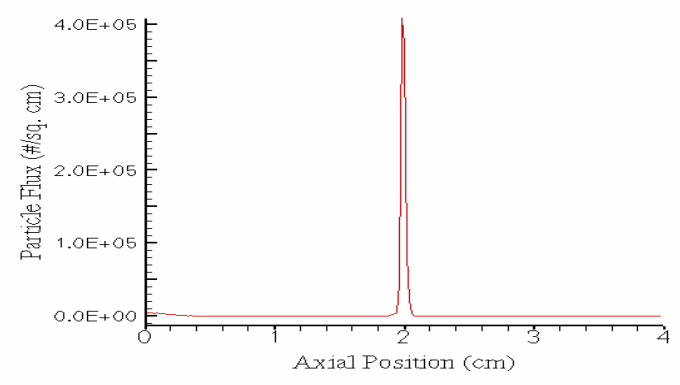

Figure 12. Primary Electrons flux profile at magnet wall of the primary electron computational domain for $15 \mathrm{~cm}$ magnet spacing

In order to better understand what is occurring in the cusp region Fig. 12 and Table 2 have been prepared. Figure 12 shows that almost all the electrons exiting the primary electron computational domain are getting absorbed in a very small area centered directly over the cusp. This area is on the order of or smaller than $0.02 \mathrm{~cm}^{2}$. This is the size of the spatial grid used in the primary electron calculations. Table 2 displays the flux of particles leaving at the center of the cusp for all the magnet spacing cases studied. These fluxes are horrendously large. Please note that the 
numbers in the table need to be multiplied by $10^{4}$. The cases with the shortest normalized confinement lengths, $z_{m}=$ $4,5,6,7$, and 8 , have the highest particle fluxes. This would indicate that these magnetic fields focus the particles at the cusp better than the other magnet spacing cases. Also displayed in the table is the number of particles leaving the computational domain outside of the wall surface that covers the magnet. Since 20,000 particles were used in this computation it can be concluded that essentially all particles leave the computational domain at the cusp. The $15 \mathrm{~cm}$ magnet spacing has the most particles leaving in the noncusp portion of the upper wall. The reason for this is that the

Table 2. Particle absorbed outside the cusp and particle flux in the cusp region.

\begin{tabular}{|c|c|c|}
\hline $\begin{array}{c}\text { Magnet } \\
\text { Spacing } \\
(\mathrm{cm})\end{array}$ & $\begin{array}{c}\text { Cusp } \\
\text { Particle Flux } \\
\left(10^{-4}, \# / \mathrm{cm}^{2}\right)\end{array}$ & $\begin{array}{c}\text { Particles } \\
\text { Absorbed } \\
\text { Outside } \\
\text { Cusp }\end{array}$ \\
\hline 3 & 47.1 & 10 \\
\hline 4 & 48.0 & 2 \\
\hline 5 & 48.2 & 0 \\
\hline 6 & 48.7 & 2 \\
\hline 7 & 48.8 & 15 \\
\hline 8 & 49.2 & 59 \\
\hline 11 & 47.0 & 409 \\
\hline 15 & 42.3 & 888 \\
\hline
\end{tabular}

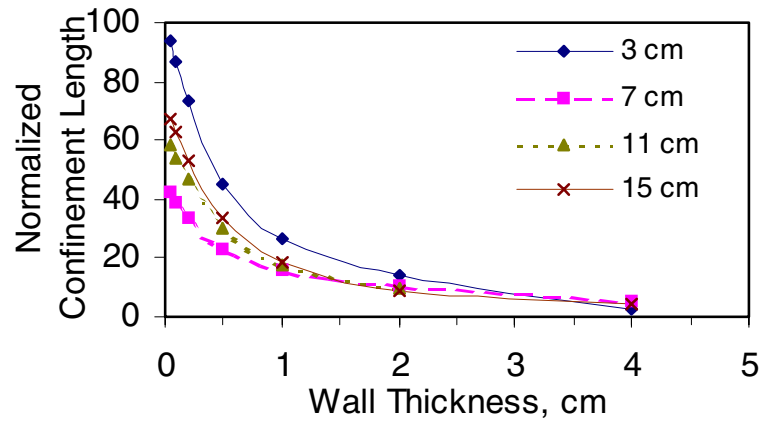

Figure 13. Effect of wall thickness on the confinement length

spacing for the magnets is too large and holes are opening between the magnets. This hole even shows up $2 \mathrm{~cm}$ out form the center magnetic. The least number of particles are leaving the computational domain outside of the cusp region for the $5 \mathrm{~cm}$ spacing case. This is the case with the shortest confinement length.

One more plot that sheds some light on the characteristics of the cusp region is shown in Fig. 13. This plot shows the variation of the confinement length with aluminum wall thickness. The aluminum has no effect on the magnetic fields, but it does move the inner surface of the absorbing wall away from the surface of the magnet. It thus moves the absorbing wall out to a location where the pinch of the magnetic field is not quite as severe. As can be seen from this plot the confinement length is very sensitive to the changes in wall thickness. Even a $1 \mathrm{~mm}$ change in the wall thickness has a significant effect on the confinement length. The thinnest wall included in Fig. 13 for all magnet spacing cases is $0.05 \mathrm{~cm}$. The next wall thickness shown is for a $0.1 \mathrm{~cm}$ wall thickness. In going from a wall thickness of $0.05 \mathrm{~cm}$ to a wall thickness of $0.1 \mathrm{~cm}$ the confinement length drops by 6 to $7 \%$. When the wall thickness increases to $0.5 \mathrm{~cm}$ the normalized confinement length drops by more than $46 \%$.

Figure 13 also shows that the ordering in the normalized confinement lengths for the 7,11, and $15 \mathrm{~cm}$ magnet spacing cases changes for a wall thickness between 1 and $2 \mathrm{~cm}$. This means that the shape of the magnet field within a radial distance of 1 to $2 \mathrm{~cm}$ of the magnet surface is what causes the confinement lengths of the 11 and $15 \mathrm{~cm}$ magnet spacing cases to produce larger confinement lengths than the $5 \mathrm{~cm}$ case. Thus this is the important region to look at for magnetic field differences. In looking directly at the field lines it is not possible to pick out differences between the shapes of the field lines in the cusp. For this reason Table 3 and Table 4 were prepared.

Table 3. $\int\left|B_{z}\right| d \ell$ across radial line above magnet surface in gauss-m.

\begin{tabular}{|c|c|c|c|c|}
\hline \multirow{2}{*}{$\begin{array}{c}\mathrm{z}_{\mathrm{m}} \\
(\mathrm{cm})\end{array}$} & \multicolumn{4}{|c|}{$\begin{array}{l}\text { Radial Distance from Surface of Magnet } \\
\qquad(\mathrm{cm})\end{array}$} \\
\hline & 0.1 & 0.2 & 0.5 & 1.0 \\
\hline 3 & 75.0 & 52.3 & 20.6 & 7.1 \\
\hline 5 & 74.5 & 51.8 & 19.9 & 6.4 \\
\hline 11 & 74.2 & 51.7 & 19.7 & 6.2 \\
\hline 15 & 74.4 & 51.6 & 19.7 & 6.2 \\
\hline
\end{tabular}

Table 3 shows the integral of the absolute value of the axial magnetic field component across a line that is parallel to the face of the magnet and as long as the face of the magnet. Table 4 shows the integral of the absolute value of the radial magnetic field component along the same line as the axial fields were integrated. These 
integrations are performed at different radial distances from the magnet surface. The line across which these integrations are performed is shown in Fig. 2. The radial distance the integration line is from the face of the magnet is shown in the first row of the tables. The magnet spacing case is shown in the first column of the table.

The axial magnetic field component is the one that keeps the primary electrons from moving in the radial direction and the radial magnetic field component is the one that keeps the primary electrons from moving in the axial direction. This means that strong axial magnetic field components should keep the primary electrons from reaching the cusp wall. From Table 3 the $3 \mathrm{~cm}$ case shows the greatest values of $\int\left|B_{z}\right| d \ell$. The values of $\int\left|B_{z}\right| d \ell$ tend to drop for cases with larger magnet spacing. For all magnet spacing cases the value of $\int B_{z} d \ell$ decreases the further from the magnet the integration is done. The values of $\int\left|B_{r}\right| d \ell$ given in Table 4 show similar trends. The authors think that larger values of $\int\left|B_{r}\right| d \ell$ may shorten the confinement length. The reason for this is that large

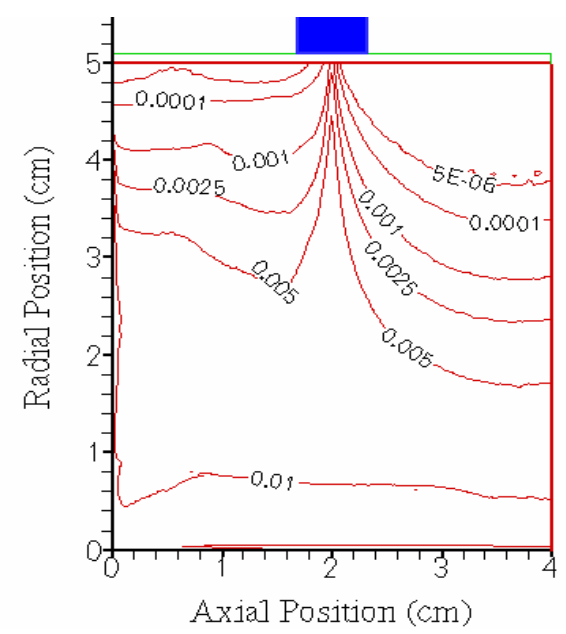

Figure 14. Relative primary electron number densities for $3 \mathrm{~cm}$ magnet spacing case.

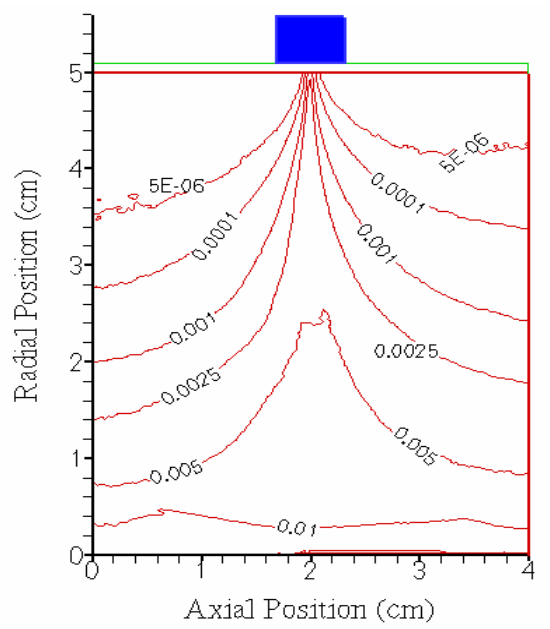

Figure 15. Relative primary electron number densities for $5 \mathrm{~cm}$ magnet spacing case. radial magnetic field components tend to focus the primary electrons into the cusp. Based on the radial magnetic field component the $3 \mathrm{~cm}$ magnet spacing is poor. Therefore there could be two contradictory trends causing the behavior seen in Fig. 11. These two competing trends could be the reason that there are high values of the normalized confinement length for the extreme spacing cases and smaller values for the middle magnet spacing cases.

Figures 14,15 , and 16 show the relative number density profiles for the $3 \mathrm{~cm}, 5 \mathrm{~cm}$, and $15 \mathrm{~cm}$ magnetic spacing cases. In all three cases the relative number densities follow the magnetic field lines. There is some asymmetry in the relative number density contours because the primary electrons are emitted on the centerline in the left corner. The greatest asymmetric behavior can be seen in the $3 \mathrm{~cm}$ magnet spacing case. The greatest primary electron number densities are close to the centerline and they tend to remain large down close to the magnet in the cusp region. This indicates that primary electrons are leaving the discharge chamber through the cusp. 


\section{B. Magnetic Field Strength}

A plot of the confinement length as a function of the magnetic field strength is shown in Fig. 17. Magnetic field strengths of $0.1,0.5,0.9,1.0,1.1,1.5$ and 2.0 times that of the permanent magnets used is utilized. The field shapes are all the same because all magnetic fields were simply scaled from the permanent magnet field for the given magnet spacing. Once again some interesting behavior is noticed. There are some dips in the

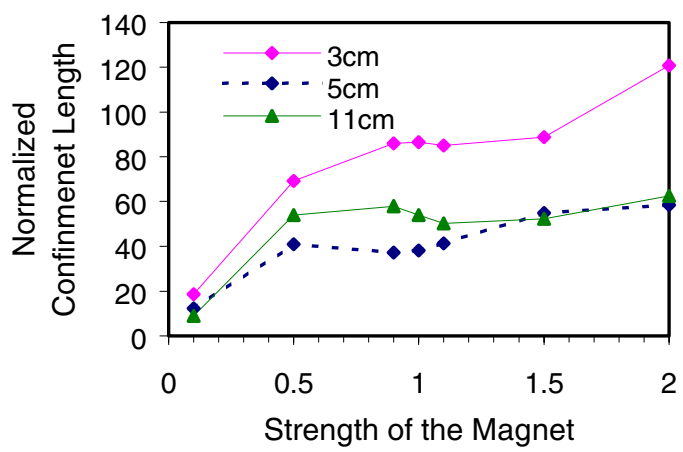

Figure 17. Effect of magnetic field strength displayed as a function of the field strength and the spacing between the magnets.

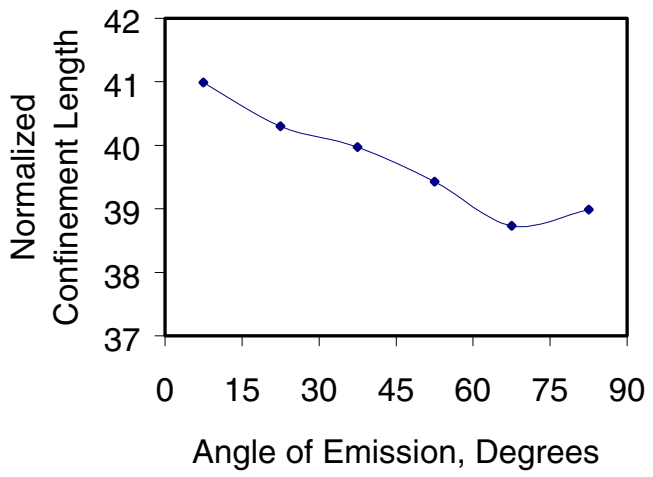

Figure 18. Effect of angle of emission for $7 \mathrm{~cm}$ spacing between the magnets.

normalized confinement length as the field strength is increased. For the $3 \mathrm{~cm}$ magnet separation case the dip occurs at slightly larger magnetic field strengths than the $5 \mathrm{~cm}$ magnet separation case. The dip in the normalized confinement length is relatively small.

\section{Primary Electrons Entrance Location}

The effect of moving the primary electron emission point from the left corner on the centerline to the center of the domain on the centerline was small. The normalized confinement length with the emission point at the left corner is 39.83 and the normalized confinement length with the emission point at the center is 37.89 . This data was determined for the $7 \mathrm{~cm}$ magnetic spacing distance

\section{Summary}

When it comes to the primary electron confinement length the cusp region is critical to the magnitude of this number. In this work a study of just the cusp region was made using a ring cusp configuration composed of three rings of permanent magnets. By varying the spacing between the three rings the shape of the magnetic field was altered. For magnet spacing from 3 to $15 \mathrm{~cm}$ the largest normalized confinement length was obtained at a magnet spacing of $3 \mathrm{~cm}$, the second largest was obtained at a magnet spacing of $15 \mathrm{~cm}$, and the shortest value was obtained at a magnet spacing of $5 \mathrm{~cm}$. It must be realized that these results only consider the cusp region of the center magnet and do not include effects of what is happening between the magnets. In regards to field strength the normalized confinement length generally increased with an increase in field strength with the exception of a small dip that occurred at varying field strengths for varying magnet spacing cases.

While very little difference in the normalized confinement length was noticed when the emission location was changed, a significant variation was observed as a function of the primary electron emission angle. The angle that gave the primary electron the most direct path into the cusp produced the shortest confinement length and the angle that shot the electron in the radial direction provided the longest confinement length. 


\section{References}

${ }^{1}$ Arakwa, Y., and Ishihara, K., "A numerical code for cusped ion thrusters," International Electric Propulsion Conference, IEPC -91-118, 1991

${ }^{2}$ Arakwa, Y., and Yamada, T., "Monte Carlo simulations of primary electron motions in cusped discharge chambers," International Electric Propulsion Conference, AIAA-90-2654, 1990.

${ }^{3}$ Mahalingam, S., and Menart, J., "Primary electron modeling in the discharge chamber of an ion engine," AIAA paper number 2002-4262, 2002.

${ }^{4}$ Hirakawa, M., and Arakawa, Y., "Plasma particle simulation in cusped ion thrusters," International Electrical Propulsion Conference, IEPC-93-242, 1993.

${ }^{5}$ Samec, T. K., "Plasma confinement of surface magnetic fields," Ph. D. Thesis, University of California, 1976.

${ }^{6}$ Griffiths, D. J., Introduction to Electrodynamics, 2nd Edition, Prentice Hall, Englewood Cliffs, New Jersey, (1989).

${ }^{7}$ Marcus, A.J., Knorr, G., and Joyce, G., "Two-dimensional simulation of cusp confinement of a plasma," Plasma Physics, Vol. 22, pp. 1015-1027, 1980.

${ }^{8}$ Leung, K.N., Hershkowitz, N., and Mackenzie, K., "Plasma confinement by localized cusps,” Physics of Fluids, Vol. 19, pp. 1045-1053, 1976

${ }^{9}$ Bosch, R.A. and Berlino, R.L., "Confinement properties of a low-beta discharge in a spindle cusp magnetic field," Physics of Fluids, Vol. 19, pp. 1045-1053, 1976.

${ }^{10}$ Ansoft Corporation, Maxwell 2D web site, http://www.ansoft.com/products/em/max2d/index.cfm, 2003.

${ }^{11}$ Patterson, M.J., Haag, T.W., and Hovan, S.A., "Performance of the NASA $30 \mathrm{~cm}$ ion thruster," IEPC paper number 93-108, 1993

${ }^{12}$ Marion, J.B., Classical Dynamics of Particles and Systems, Academic Press, NY, 1970.

${ }^{13}$ Greenwood, D.T., Classical Dynamics, Prentice-Hall, Englewood Cliffs, NJ, 1997

${ }^{14}$ Brophy, J.R., and Wilbur, P.J., "Simple performance model for ring and line cusp ion thruster," AIAA Journal, Vol. 23, pp. $1731-1736,1985$.

${ }^{15}$ Hiatt, J. and Wilbur, P., "Ring cusp discharge chamber performance optimization," International Electric Propulsion Conference, AIAA-85-2007, 1985 
Public reporting burden for this collection of information is estimated to average 1 hour per response, including the time for reviewing instructions, searching existing data sources, gathering and maintaining the data needed, and completing and reviewing the collection of information. Send comments regarding this burden estimate or any other aspect of this collection of information, including suggestions for reducing this burden, to Washington Headquarters Services, Directorate for Information Operations and Reports, 1215 Jefferson Davis Highway, Suite 1204, Arlington, VA 22202-4302, and to the Office of Management and Budget, Paperwork Reduction Project (0704-0188), Washington, DC 20503.

\begin{tabular}{|l|l|l}
\hline 1. AGENCY USE ONLY (Leave blank) & $\begin{array}{c}\text { 2. REPORT DATE } \\
\text { August } 2004\end{array}$ & $\begin{array}{c}\text { 3. REPORT TYPE AND DATES COVERED } \\
\text { Final Contractor Report }\end{array}$ \\
\hline
\end{tabular}

4. TITLE AND SUBTITLE

5. FUNDING NUMBERS

Computational Study of Primary Electrons in the Cusp Region of an Ion Engine's

Discharge Chamber

6. AUTHOR(S)

Shirin S. Deshpande, Sudhakar Mahalingam, and James A. Menart

WBS-22-319-20-B2

NAG3-2907

7. PERFORMING ORGANIZATION NAME(S) AND ADDRESS(ES)

8. PERFORMING ORGANIZATION

REPORT NUMBER

Wright State University

3640 Colonel Glenn

E-14709

Dayton, Ohio 45435-0002

10. SPONSORING/MONITORING

9. SPONSORING/MONITORING AGENCY NAME(S) AND ADDRESS(ES)

National Aeronautics and Space Administration

Washington, DC 20546-0001

AGENCY REPORT NUMBER

NASA CR-2004-213200

AIAA-2004-4109

\section{SUPPLEMENTARY NOTES}

Prepared for the 40th Joint Propulsion Conference and Exhibit cosponsored by the AIAA, ASME, SAE, and ASEE, Fort Lauderdale, Florida, July 11-14, 2004. Project Manager, Thomas J. Stueber, Power and On-Board Propulsion

Technology Division, NASA Glenn Research Center, organization code 5430, 216-433-2218.

12a. DISTRIBUTION/AVAILABILITY STATEMENT

12b. DISTRIBUTION CODE

Unclassified - Unlimited

Subject Category: 75

Distribution: Nonstandard

Available electronically at http://gltrs.grc.nasa.gov

This publication is available from the NASA Center for AeroSpace Information, 301-621-0390.

13. ABSTRACT (Maximum 200 words)

In this work a computer code called PRIMA is used to study the motion of primary electrons in the magnetic cusp region of the discharge chamber of an ion engine. Even though the amount of wall area covered by the cusps is very small, the cusp regions are important because prior computational analyses have indicated that most primary electrons leave the discharge chamber through the cusps. The analysis presented here focuses on the cusp region only. The affects of the shape and size of the cusp region on primary electron travel are studied as well as the angle and location at which the electron enters the cusp region. These affects are quantified using the confinement length and the number density distributions of the primary electrons. In addition to these results comparisons of the results from PRIMA are made to experimental results for a cylindrical discharge chamber with two magnetic rings. These comparisons indicate the validity of the computer code called PRIMA.

\section{SUBJECT TERMS}

Ion engines; Propulsion; Plasmas (physics)

\begin{tabular}{|c|c|c|}
\hline $\begin{array}{c}\text { 17. SECURITY CLASSIFICATION } \\
\text { OF REPORT } \\
\text { Unclassified }\end{array}$ & $\begin{array}{c}\text { 18. SECURITY CLASSIFICATION } \\
\text { OF THIS PAGE } \\
\text { Unclassified }\end{array}$ & $\begin{array}{c}\text { 19. SECURITY CLASSIFICATION } \\
\text { OF ABSTRACT } \\
\text { Unclassified }\end{array}$ \\
\hline
\end{tabular}

NSN 7540-01-280-5500

Standard Form 298 (Rev. 2-89) 

